\title{
Towards the Distribution of the Size of a Largest Planar Matching and Largest Planar Subgraph in Random Bipartite Graphs
}

\author{
MARCOS KIWI* \\ Depto. Ing. Matemática and \\ Ctr. Modelamiento Matemático (UMI 2807, CNRS) \\ University of Chile \\ Correo 3, Santiago 170-3, Chile \\ e-mail: mkiwi@dim.uchile.cl
}

\author{
MARTIN LOEBL ${ }^{\dagger}$ \\ Dept. of Applied Mathematics and \\ Institute of Theoretical Computer Science (ITI) \\ Charles University \\ Malostranské nám. 25, 11800 Praha 1 \\ Czech Republic \\ e-mail: loebl@kam.mff.cuni.cz
}

Submitted: May 31, 2007; Accepted: Oct 16, 2008; Published: Oct 20, 2008

Mathematics Subject Classification: 05A15

\begin{abstract}
We address the following question: When a randomly chosen regular bipartite multigraph is drawn in the plane in the "standard way", what is the distribution of its maximum size planar matching (set of non-crossing disjoint edges) and maximum size planar subgraph (set of non-crossing edges which may share endpoints)? The problem is a generalization of the Longest Increasing Sequence (LIS) problem (also called Ulam's problem). We present combinatorial identities which relate the number of $r$-regular bipartite multigraphs with maximum planar matching (maximum planar subgraph) of at most $d$ edges to a signed sum of restricted lattice walks in $\mathbb{Z}^{d}$, and to the number of pairs of standard Young tableaux of the same shape and with a "descend-type" property. Our results are derived via generalizations of two combinatorial proofs through which Gessel's identity can be obtained (an identity that is crucial in the derivation of a bivariate generating function associated to the distribution of the length of LISs, and key to the analytic attack on Ulam's problem). Finally, we generalize Gessel's identity. This enables us to count, for small values of $d$ and $r$, the number of $r$-regular bipartite multi-graphs on $n$ nodes per color class with maximum planar matchings of size $d$. Our work can also be viewed as a first step in the study of pattern avoidance in ordered bipartite multi-graphs.
\end{abstract}

Keywords: Gessel's identity, longest increasing sequence, random bipartite graphs, lattice walks.

\footnotetext{
${ }^{*}$ Gratefully acknowledges the support of MIDEPLAN via ICM-P01-05, and CONICYT via FONDECYT 1010689, FONDAP in Applied Mathematics, and Anillo en Redes ACT08.

Gratefully acknowledges the support of ICM-P01-05. This work was done while visiting the Depto. Ing. Matemática, U. Chile.
} 


\section{Introduction}

Let $U$ and $V$ henceforth denote two disjoint totally ordered sets (both order relations will be referred to by $\preceq$ ). Typically, we will consider the case where $|U|=|V|=n$ and denote the elements of $U$ and $V$ by $u_{1} \preceq u_{2} \preceq \ldots \preceq u_{n}$ and $v_{1} \preceq v_{2} \preceq \ldots \preceq v_{n}$ respectively.

Let $G=(U, V ; E)$ denote a bipartite multi-graph with color classes $U$ and $V$. Two distinct edges $e$ and $e^{\prime}$ of $G$ such that $e=u v$ and $e^{\prime}=u^{\prime} v^{\prime}$ are said to be noncrossing if $u \neq u^{\prime}, v \neq v^{\prime}$, and $u$ and $u^{\prime}$ are in the same order as $v$ and $v^{\prime}$; in other words, if $u \prec u^{\prime}$ and $v \prec v^{\prime}$ or $u^{\prime} \prec u$ and $v^{\prime} \prec v$. A matching of $G$ is called planar if every distinct pair of its edges is noncrossing. We let $L(G)$ denote the number of edges of a maximum size (largest) planar matching in $G$ (note that $L(G)$ depends on the graph $G$ and on the ordering of its color classes). For the sake of simplicity we will concentrate solely in the case where $|E|=r n$ and $G$ is $r$-regular.

When $r=1$, an $r$-regular multi-graph with color classes $U$ and $V$ may be naturally identified with a permutation. A planar matching becomes an increasing sequence of the permutation, where an increasing sequence of length $L$ of a permutation $\pi$ of $\{1, \ldots, n\}$ is a sequence $1 \leq i_{1}<i_{2}<\ldots<i_{L} \leq n$ such that $\pi\left(i_{1}\right)<\pi\left(i_{2}\right)<\ldots<\pi\left(i_{L}\right)$. The Longest Increasing Sequence (LIS) problem concerns the determination of the asymptotic, on $n$, behavior of the length of a LIS for a randomly and uniformly chosen permutation $\pi$. The LIS problem is also referred to as "Ulam's problem" (e.g., in [Kin73, BDJ99, Oko00]). Ulam is often credited for raising it in [Ula61] where he mentions (without reference) a "well-known theorem" asserting that given $n^{2}+1$ integers in any order, it is always possible to find among them a monotone subsequence of $n+1$ (the theorem is due to Erdős and Szekeres [ES35]). Monte Carlo simulations are reported in [BB67], where it is observed that over the range $n \leq 100$, the limit $\gamma$ of the LIS of $n^{2}+1$ randomly chosen elements approaches 2 when normalized by $n$. Hammersley [Ham72] gave a rigorous proof of the existence of the limit $\gamma$ and conjectured it was equal to 2. Later, Logan and Shepp [LS77], based on a result by Schensted [Sch61], proved that $\gamma \geq 2$; finally, Vershik and Kerov [VK77] obtained that $\gamma \leq 2$. In a major recent breakthrough due to Baik, Deift, and Johansson [BDJ99] the asymptotic distribution of the LIS was determined. For a detailed account

of these results, history and related work see the surveys of Aldous and Diaconis [AD99] and Stanley [Sta02].

\subsection{Main Results}

In this article we establish combinatorial identities which express the number of $r$-regular bipartite multi-graphs with planar matchings with at most $d$ edges in terms of:

- A signed sum of restricted lattice walks in $\mathbb{Z}^{d}$ (Theorem 7).

- The number of pairs of standard Young tableaux of the same shape and with a "descendtype" property (Theorem 10).

Our arguments can be adapted in order to characterize the distribution of the largest size of planar subgraphs of randomly chosen $r$-regular bipartite multi-graphs (Theorem 9). 
Let $g_{r}(n ; d)$ denote the number of $r$-regular bipartite multi-graphs on $n$ nodes per color class with planar matchings of size at most $d$. The combinatorial identities we derive allow us to show that for $r$ fixed and $d=2$, the generating function for $g_{r}(n ; d)$ is in fact algebraic (Theorem 16). For small values of $r$, we determine the first terms of such generating functions. For the case where $d>2$ and $r$ and $n$ are small, our results allow us to explicitly compute $g_{r}(n ; d)$ (Table 1). Finally, in Theorem 21 we express the generating function of $g_{r}(n ; d)$ ( $r$ and $d$ fixed) as a differential operator applied to a determinant. This generalizes Gessel's identity.

Our results concern enumeration of pattern avoiding ordered graphs. Pattern avoidance in permutations is an extensively studied subject. See [Bó4, Ch. 4] for a more in depth discussion of the pattern avoidance area. Other results of enumerative character on pattern avoidance in ordered graphs may be found in $\left[\mathrm{CDD}^{+} 07, \mathrm{BR} 01\right.$, de 07]. For recent results of enumerative type on restricted lattice paths see [BF02, BM06].

\subsection{Acknowledgement}

We would like to thank an anonymous referee for several suggestions which significantly improved the presentation of the paper. In particular, Section 4 is an elaboration on her/his suggestions.

\subsection{Models of Random Graphs: From $k$-regular Multi-graphs to Permu- tations}

Most work on random regular graphs is based on the so called random configuration model of Bender and Canfield and Bollobás [Bol85, Ch. II, § 4]. Below we follow this approach, but first we need to adapt the configuration model to the bipartite graph scenario. Given $U, V, n$ and $r$ as above, let $\bar{U}=U \times[r]$ and $\bar{V}=V \times[r]$. An $r$-configuration of $U$ and $V$ is a one-to-one

pairing of $\bar{U}$ and $\bar{V}$. Hence, a configuration will be naturally identified with the corresponding permutation from $\bar{U}$ to $\bar{V}$.

The natural projection of $\bar{U}=U \times[r]$ and $\bar{V}=V \times[r]$ onto $U$ and $V$ respectively (ignoring the second coordinate) projects each permutation $F$ to a bipartite multi-graph $\pi(F)$ with color classes $U$ and $V$. Note in particular that $\pi(F)$ may contain multiple edges (arising from sets of two or more edges in $F$ whose end-points correspond to the same pair of vertices in $U$ and $V$ ). However, the projection of the uniform distribution over the permutations is not the uniform distribution over all $r$-regular bipartite multi-graphs on $U$ and $V$ (the probability of obtaining a given multi-graph is proportional to the product of factors $1 / j$ ! for each multiple edge of multiplicity $j$ ). Since a permutation $F$ can be considered an ordered 1-regular graph, it makes perfect sense to speak of the size $L(F)$ of its largest planar matching.

We denote an element $(u, i) \in \bar{U}$ by $u^{i}$ and adopt an analogous convention for the elements of $\bar{V}$. We shall abuse notation and denote by $\preceq$ the total order on $\bar{U}$ given by $u^{i} \preceq \widetilde{u}^{j}$ if $u \prec \widetilde{u}$ or $u=\widetilde{u}$ and $i \leq j$. We adopt a similar convention for $\bar{V}$.

Let $G_{r}(U, V ; d)$ denote the set of all $r$-regular bipartite multi-graphs on $U$ and $V$ whose largest planar matching is of size at most $d$. Note that if $|U|=|V|=n$, then the cardinality of $G_{r}(U, V ; d)$ depends on $U$ and $V$ solely through $n$. Unless specified otherwise, we assume 


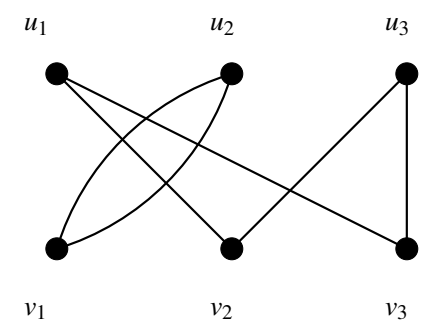

(a)

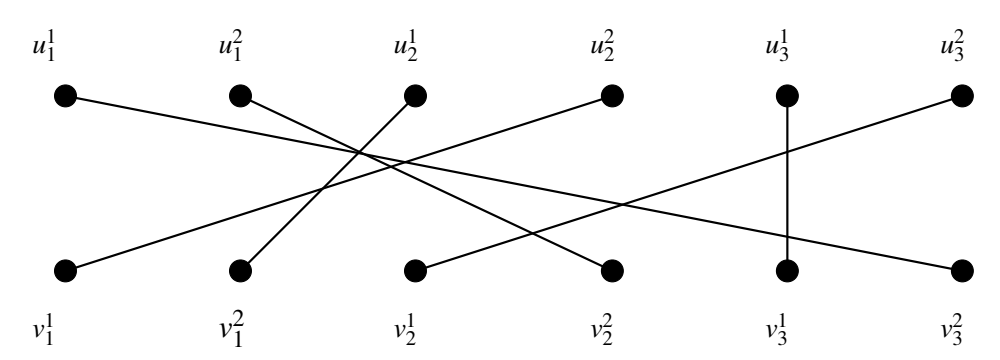

(b)

Figure 1: (a) A 2-regular ordered multi-graph $G$, and (b) the permutation $\pi$ associated to $G$.

$|U|=|V|=n$. Recall that $g_{r}(n ; d)$ denotes the number of $r$-regular multi-graphs on $n$ nodes per color class with planar matchings of size at most $d$, i.e. $g_{r}(n ; d)=\left|G_{r}(U, V ; d)\right|$.

The first step in our considerations is an identification of $G_{r}(U, V ; d)$ with a subset of permutations of $[r n]$. It is achieved by the following definition and a straightforward observation.

Definition 1. Let $S(r, n, d)$ be the subset of permutations $\pi$ of $[r n]$ which satisfy the following two conditions:

1. For all $1 \leq s<r$ and $0 \leq i<n, \pi(r i+s)>\pi(r i+s+1)$ and symmetrically $\pi^{-1}(r i+s)>$ $\pi^{-1}(r i+s+1)$,

2. The length of a LIS of $\pi$ is at most $d$.

Lemma 2. Let $|U|=|V|=n$. There is a bijection between $G_{r}(U, V ; d)$ and $S(r, n, d)$.

Several of the concepts introduced in this section are illustrated in Figure 1.

\subsection{Young tableaux}

A (standard) Young tableau of $m$ of shape $\lambda=\left(\lambda_{1}, \ldots, \lambda_{r}\right)$, where $\lambda_{1} \geq \lambda_{2} \geq \ldots \geq \lambda_{r} \geq 0$ and $m=\lambda_{1}+\ldots+\lambda_{r}$, is an arrangement $T$ of the elements of $[m]$ in an array of left-justified rows, with $\lambda_{i}$ elements in row $i$, such that the entries in each row are in increasing order from left to right, and the entries of each column are increasing from top to bottom (here we follow the 
usual convention that considers row $i$ to be above row $i+1)$. One says that $T$ has $r$ rows and $c$ columns if $\lambda_{r}>0$ and $c=\lambda_{1}$ respectively.

The Robinson correspondence (rediscovered independently by Schensted) shows that the set of permutations of $[m]$ is in one to one correspondence with the collection of pairs of equal shape Young tableaux of $m$. The correspondence can be constructed through the RobinsonSchensted-Knuth (RSK) algorithm — also referred to as row-insertion or row-bumping algorithm. The algorithm takes a tableau $T$ and a positive integer $x$, and constructs a new tableau, denoted $T \leftarrow x$. This tableau will have one more box than $T$, and its entries will be those of $T$ together with one more entry labeled $x$, but there is some moving around, the details of which are not of direct concern to us, except for the following fact:

Lemma 3. [Bumping Lemma [Ful97, pag. 9]] Consider two successive row-insertions, first row inserting $x$ in a tableau $T$ and then row-inserting $x^{\prime}$ in the resulting tableau $T \leftarrow x$. This gives rise to two new boxes $B$ and $B^{\prime}$ containing $x$ and $x^{\prime}$ as shown in Figure 2:

- If $x \leq x^{\prime}$, then $B$ is strictly left of and weakly below $B^{\prime}$.

- If $x>x^{\prime}$, then $B^{\prime}$ is weakly left of and strictly below $B$.

Given a permutation $\pi$ of $[m]$, the RSK algorithm associates to $\pi$ a pair $(P(\pi), Q(\pi))$ of Young tableaux of $m$ of the same shape by,

- sequentially row inserting $\pi(1), \ldots, \pi(m)$ to an initially empty pair of tableaux, and thus obtaining $P(\pi)$, and,

- placing the value $i$ into the box of $Q(\pi)$ 's diagram corresponding to the box created during the $i$-th insertion into $P(\pi)$.

Two remarkable facts about the RSK algorithm which we will exploit are:

Theorem 4. [RSK Correspondence [Ful97, pag. 40]] The RSK correspondence sets up a oneto-one mapping between permutations of $[m]$ and pairs of Young tableaux $(P, Q)$ of $m$ and with the same shape.

Theorem 5. [Symmetry Theorem [Ful97, pag. 40]] If $\pi$ is a permutation of $[m]$, then $P\left(\pi^{-1}\right)=$ $Q(\pi)$ and $Q\left(\pi^{-1}\right)=P(\pi)$.

The following corollary of Lemma 3 and Theorem 4 is credited to Schensted (see [Ful97]).

Corollary 6. Let $\pi$ be a permutation of $[\mathrm{m}]$. Then, $\pi$ has no ascending sequence of length greater than $d$ if and only if $P(\pi)$ and $Q(\pi)$ have at most $d$ columns.

The reader interested in an in depth discussion of Young tableaux is referred to [Ful97]. 


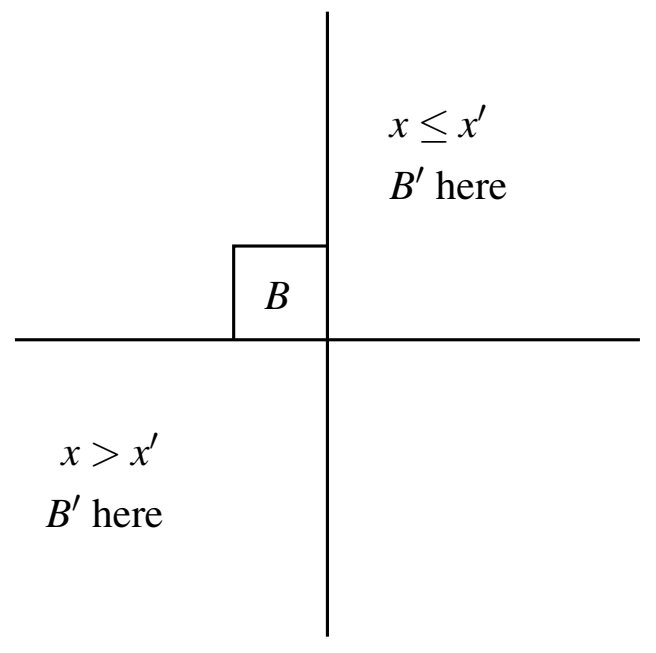

Figure 2: New tableau entries created through row-insertions.

\subsection{Walks}

We say that $w=w_{0} \ldots w_{m}$ is a lattice walk in $\mathbb{Z}^{d}$ of length $m$ if $\left\|w_{i}-w_{i-1}\right\|_{1}=1$ for all $1 \leq i \leq m$. Moreover, we say that $w$ starts at the origin and ends in $\vec{p}$ if $w_{0}=\overrightarrow{0}$ and $w_{m}=\vec{p}$. For the rest of this paper, all walks are to be understood as lattice walks in $\mathbb{Z}^{d}$. Henceforth, let $W(d, r, m ; \vec{p})$ denote the set of all walks of length $m$ from the origin to $\vec{p} \in \mathbb{Z}^{d}$.

We will identify the walk $w=w_{0} \cdots w_{m}$ with the sequence $d_{1} \ldots d_{m}$ such that $w_{i}-w_{i-1}=$ $\operatorname{sign}\left(d_{i}\right) \vec{e}_{\left|d_{i}\right|}$, where $\vec{e}_{j}$ denotes the $j$-th element of the canonical basis of $\mathbb{Z}^{d}$. If $d_{i}$ is negative, then we say that the $i$-th step is a negative step in direction $\left|d_{i}\right|$, or negative step for short. We adopt a similar convention when $d_{i}$ is positive.

We say that two walks are equivalent if both subsequences of the positive and the negative steps are the same. For each equivalence class consider the representative for which the positive steps precede the negative steps. Each such representative walk may hence be written as $a_{1} a_{2} \cdots \mid b_{1} b_{2} \cdots$ where the $a_{i}$ 's and $b_{j}$ 's are all positive. For an arbitrary collection of walks $W$, we henceforth denote by $W^{*}$ the collection of the representative walks in $W$.

Recall that one can associate to a permutation $\pi$ of $[d]$ the Toeplitz point $T(\pi)=(1-$ $\pi(1), \ldots, d-\pi(d))$. Note that in a walk from the origin to a Toeplitz point, the number of steps in a positive direction equals the number of steps in a negative direction. In particular, each such walk has an even length.

In cases where we introduce notation for referring to a family of walks from the origin to a given lattice point $\vec{p}$, such as $W(d, r, m ; \vec{p})$, we sometimes consider instead of $\vec{p}$ a subset of lattice points $P$. It is to be understood that we are thus making reference to the set of all walks in the family that end at a point in $P$. A set of lattice points of particular interest to the ensuing discussion is the set of Toeplitz points, henceforth denoted by $\mathbb{T}$.

When $m=r n$ the sequences of positive and negative steps in a walk in $W(d, r, 2 m ; \mathbb{T})$ will 
be referred to as:

$$
a_{u_{1}^{1}} \cdots a_{u_{1}^{r}} a_{u_{2}^{1}} \cdots a_{u_{2}^{r}} \cdots a_{u_{n}^{1}} \cdots a_{u_{n}^{r}} \quad \text { and } \quad b_{v_{1}^{1}} \cdots b_{v_{1}^{r}} b_{v_{2}^{1}} \cdots b_{v_{2}^{r}} \cdots b_{v_{n}^{1}} \cdots b_{v_{n}^{r}}
$$

In the ensuing discussion we will associate elements of $G_{r}(U, V ; d)$ to walks in $W^{*}(d, r, 2 m ; \mathbb{T})$ whose positive steps $a_{u_{1}^{1}} \cdots a_{u_{n}^{r}}$ and negative steps $b_{v_{1}^{1}} \cdots b_{v_{n}^{r}}$ satisfy:

Condition (I): $a_{u^{i}} \geq a_{u^{i+1}}$ and $b_{v^{i}} \geq b_{v^{i+1}}$ for all $u \in U, v \in V$ and $1 \leq i<r$.

The collection of walks in $W^{*}(d, r, 2 m ; T(\pi))$ which respect condition (I) will be denoted by $W_{I}^{*}(d, r, 2 m ; T(\pi))$.

\section{Avoiding Large Planar Matchings and Large Planar Sub- graphs}

We are now ready to state the first result of the paper.

Theorem 7. For all positive integers $n, d, r$,

$$
g_{r}(n ; d)=\sum_{\pi \in S_{d}} \operatorname{sign}(\pi)\left|W_{I}^{*}(d, r, 2 r n ; T(\pi))\right|
$$

The case $r=1$ is proved in [GWW98] where it is used to give a combinatorial proof of Gessel's Identity [Ges90]:

Theorem 8. [Gessel's Identity] Let $I_{v}(x)$ denote the Bessel function of imaginary argument, i.e.

$$
I_{v}(x)=\sum_{m \geq 0} \frac{1}{m ! \Gamma(m+v+1)}\left(\frac{x}{2}\right)^{2 m+v} .
$$

Then,

$$
\sum_{m \geq 0} \frac{g_{1}(m ; d)}{m !^{2}} x^{2 m}=\operatorname{det}\left(I_{|r-s|}(2 x)\right)_{r, s=1, \ldots, d} .
$$

In the next section we give a sketch and a proof of Theorem 7. Both are straightforward adaptations of the proofs in [GWW98] dealing with the $r=1$ case.

In order to motivate the second main result of this work we describe a random process which researchers have studied, either explicitly or implicitly, in several different contexts. Let $X_{i, j}$ be a non-negative random variable associated to the lattice point $(i, j) \in[n]^{2}$. For $C \subseteq$ $[n]^{2}$, we refer to $\sum_{(i, j) \in C} X_{i, j}$ as the weight of $C$. We are interested in the determination of the distribution of the maximum weight of $C$ over all $C=\left\{\left(i_{1}, j_{1}\right),\left(i_{2}, j_{2}\right), \ldots\right\}$ such that $i_{1}, i_{2}, \ldots$ and $j_{1}, j_{2}, \ldots$ are strictly increasing. Johansson [Joh00] considered the case where the $X_{i, j}$ 's are independent identically distributed according to a geometric distribution. Sepäläinen [Sep77] and Gravner, Tracy, and Widom [GTW01] studied the case where the $X_{i, j}$ 's are independent identically distributed Bernoulli random variables (but, in the latter paper, the collections of 
lattice points $C=\left\{\left(i_{1}, j_{1}\right),\left(i_{2}, j_{2}\right), \ldots\right\}$ were such that $i_{1}, i_{2}, \ldots$ and $j_{1}, j_{2}, \ldots$ were weakly and strictly increasing respectively).

Our Theorem 7 concerns the case of $\left(X_{i, j}\right)_{(i, j) \in[n]^{2}}$ uniformly distributed over all adjacency matrices of $r$-regular multi-graphs. A natural question is whether a similar result holds if one relaxes the requirement that the sequences $i_{1}, i_{2}, \ldots$ and $j_{1}, j_{2}, \ldots$ are strictly increasing. For example, if one allows them to be weakly increasing. This is equivalent to asking for the distribution of the size of a largest planar subgraph, i.e. the largest set of non-crossing edges which may share endpoints, in a uniformly chosen $r$-regular multigraph. The same line of argument that we will use in the derivation of Theorem 7 yields:

Theorem 9. Let $\hat{g}_{r}(n ; d)$ be the number of r-regular bipartite multi-graphs on $n$ nodes per color class with no planar subgraph of more than d edges. Let $\widehat{W}(d, r, 2 r n ; T(\pi))$ be the set of all walks in $W^{*}(d, r, 2 r n ; T(\pi))$ whose positive steps $a_{u_{1}^{1}} \cdots a_{u_{n}^{r}}$ and negative steps $b_{v_{1}^{1}} \cdots b_{v_{n}^{r}}$ satisfy: $a_{u^{i}}<a_{u^{i+1}}$ and $b_{v^{i}}<b_{v^{i+1}}$ for all $u \in U, v \in V$ and $1 \leq i<r$. Then,

$$
\hat{g}_{r}(n ; d)=\sum_{\pi \in S_{d}} \operatorname{sign}(\pi)|\widehat{W}(d, r, 2 r n ; T(\pi))|
$$

\section{A proof of Theorem 7}

First, we can write a few lines proof by simply pointing out that the mapping $\phi$ of Section 3 of [GWW98], when restricted to $S(r, n, d)$, is into $W_{I}^{*}(d, r, 2 m ; \overrightarrow{0})$. The parity reversing involution of the same section of [GWW98] induces a parity reversing involution on $W_{I}^{*}(d, r, 2 m ; \mathbb{T}) \backslash$ $\phi(S(r, n, d))$. This suffices to prove Theorem 7. Theorem 9 follows in the same way; one needs to reverse the two ' $>$ ' in the definition of $S(r, n, d)$.

We chose to write another proof along the lines of the one in Section 4 of [GWW98] since along its way it also characterizes the image of $S(r, n, d)$ through the RSK correspondence.

Let $m=r n$. The following theorem follows from Lemma 3 and Theorem 5.

Theorem 10. The RSK correspondence establishes a one-to-one correspondence between the set $S(r, n, d)$ and the collection of pairs of equal shape Young tableaux of $m$ both satisfying the following property:

Condition (II): For each $i \in[n]$ and $1 \leq s<r$, the row containing $r(i-1)+s$ is strictly above the row containing $r(i-1)+s+1$.

Figure 3 illustrates the above theorem.

Example 11. Note that condition (II), as guaranteed by Theorem 10, is reflected in the tableaux shown in Figure 3 (in both tableaux 1, 3 and 5 are strictly above 2, 4 and 6 respectively).

We now describe a well-known way of associating walks to Young tableaux which to the best of our knowledge was introduced in [GWW98]. Let $T(m, d)$ denote the set of the Young tableaux of $m$ where the first row has length at most $d$. Let $\varphi$ be the mapping from $T(m, d)$ to walks in $W\left(d, r, m ; \mathbb{Z}^{d}\right)$ such that $\varphi(T)=a_{1} \cdots a_{m}$ where $a_{i}$ equals the column in which entry $i$ appears in $T$. It follows that: 

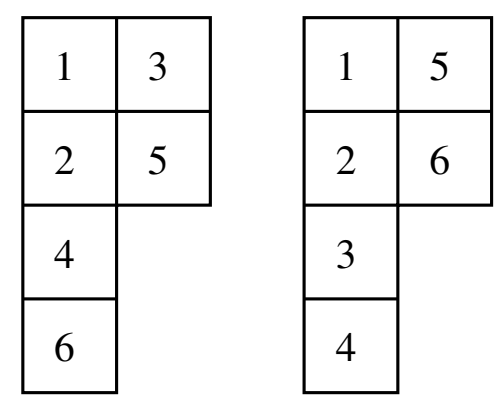

Figure 3: Pair of Young tableaux associated through the RSK algorithm to the permutation of Figure 1.b.

Lemma 12. The mapping $\varphi$ is a bijection between tableaux in $T(m, d)$ satisfying condition (II) and walks of length $m$ starting at the origin, moving only in positive directions, staying in the region $x_{1} \geq x_{2} \geq \cdots \geq x_{d}$ and satisfying condition (I).

Proof. If $\varphi(T)=\varphi\left(T^{\prime}\right)$ for $T, T^{\prime} \in T(m, d)$, then $T$ and $T^{\prime}$ have the same elements in each of their columns. Since in a Young tableau the entries of each column are increasing from top to bottom, it follows that $T=T^{\prime}$. We have thus established that $\varphi$ is an injection.

Assume now that $w=a_{1} \cdots a_{m}$ is a walk with the required properties. Denote by $C(l)$ the set of indices $j$ for which $a_{j}=l$. Note that since $w$ is a walk in $\mathbb{Z}^{d}, C(l)$ is empty for all $l>d$. Let $T$ be the tableau whose $l$-th column entries correspond to $C(l)$ (obviously ordered increasingly from top to bottom). Since $w$ stays in the region $x_{1} \geq x_{2} \geq \cdots \geq x_{d}$, we get that $|C(1)| \geq|C(2)| \geq \ldots \geq|C(d)|$ and that the entries of each row of $T$ are strictly increasing. It follows that $T$ is indeed a Young tableau.

We claim that $T$ satisfies condition (II). Indeed, let $i \in[n]$ and $1 \leq s<r$. Since $w_{r(i-1)+s} \geq$ $w_{r(i-1)+s+1}$, the entry $r(i-1)+s$ of $T$ ends in a column with an index at least as high as the one of entry $r(i-1)+s+1$. However, entries in a Young tableau are strictly increasing top to bottom and left to right. It follows that in $T$ the row containing entry $r(i-1)+s$ is strictly above the row containing entry $r(i-1)+s+1$.

Note that if $T$ and $T^{\prime}$ belong to $T(m, d)$ and have the same shape, then $\varphi(T)$ and $\varphi\left(T^{\prime}\right)$ are walks that terminate at the same lattice point.

For a walk $w=a_{1} \cdots a_{m} \mid b_{1} \cdots b_{m}$ in $W_{I}^{*}\left(d, r, 2 m ; \mathbb{Z}^{d}\right)$ we denote by $\widetilde{w}$ the walk obtained by reversing the subsequence of $w$ 's negative steps. Denote by $\widetilde{W}_{I}^{*}(d, r, 2 m ; \vec{p})$ the collection of all $\widetilde{w}$ for which $w$ belongs to $W_{I}^{*}(d, r, 2 m ; \vec{p})$.

Theorem 13. There is a bijection between $S(r, n, d)$ and the walks in $\widetilde{W}_{I}^{*}(d, r, 2 m ; \overrightarrow{0})$ staying in the region $x_{1} \geq x_{2} \geq \ldots \geq x_{d}$.

Proof. Theorem 10 and Lemma 12 give a bijection between $S(r, n, d)$ and pairs of walks of length $m$ starting at the origin, terminating at the same lattice point, moving only in positive directions, staying in the region $x_{1} \geq x_{2} \geq \ldots \geq x_{d}$ and satisfying condition (I). Say such pair of walks are $c_{1} \cdots c_{m}$ and $c_{1}^{\prime} \cdots c_{m}^{\prime}$ respectively. Then, $c_{1} \cdots c_{m} \mid c_{m}^{\prime} \cdots c_{1}^{\prime}$ is the sought after walk with the desired properties. 


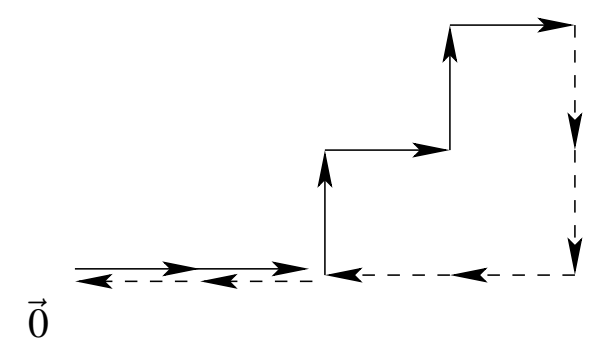

Figure 4: Walk in $\widetilde{W}_{I}^{*}(2,2,12 ; \overrightarrow{0})$ associated to the pair of Young tableaux of Figure 3 (and thus also to the graph of Figure 1).

Figure 4 illustrates the bijection of Theorem 13.

Proof. (of Theorem 7) The desired conclusion is an immediate consequence of Theorem 13 and the existence of a parity-reversing involution $\rho$ on the walks $w$ in $\widetilde{W}_{I}^{*}(d, r, 2 m ; \mathbb{T})$ that do not stay in the region $x_{1} \geq x_{2} \geq \ldots \geq x_{d}$. This involution is the same as in [GWW98] but applied to a subset of walks. Hence for the purpose of clarity of presentation we chose here to define it explicitly. The involution is most easily described if we translate the walks to start at $(d-1, d-2, \ldots, 0)$; the walks are then restricted not to lie completely in the region $R$ defined by $x_{1}>x_{2}>\ldots>x_{d}$. Let $N$ be the subset of the translated walks of $\widetilde{W}_{I}^{*}(d, r, 2 m ; \mathbb{T})$ that do not stay completely in $R$. Let $w=c_{1} \ldots c_{2 m} \in N$ and let $t$ be the smallest index such that the walk given by the initial segment $c_{1} \ldots c_{t}$ of $w$ terminates in a vertex $\left(p_{1}, \ldots, p_{d}\right) \notin R$. Hence, there is exactly one $j$ such that $p_{j}=p_{j+1}$. Observe that $c_{t}=j+1$ if $t \leq m$, and $c_{t}=j$ otherwise.

Walk $\rho(w)$ is constructed as follows:

- Leave segment $c_{1} \ldots c_{t}$ unchanged.

- For $i \in[2 n]$, let $S(i)=\{s \in[2 m]: r(i-1)<s \leq r i\}, S_{0}(i)=\left\{s \in S(i): s>t, c_{s}=j\right\}$ and $S_{1}(i)=\left\{s \in S(i): s>t, c_{s}=j+1\right\}$. For $i \leq n$ (respectively $i>n$ ), assign the value $j+1$ to the $\left|S_{0}(i)\right|$ first (respectively last) coordinates of $\left(c_{s}: s \in S_{0}(i) \cup S_{1}(i)\right)$ and the value $j$ to the remaining $\left|S_{1}(i)\right|$ coordinates.

Clearly, if $w$ terminates in $\left(q_{1}, \ldots, q_{d}\right)$, then $\rho(w)$ terminates in $\left(q_{1}, \ldots, q_{j+1}, q_{j}, \ldots, q_{d}\right)$. Hence, $\rho$ reverses the parity of $w$. Moreover, $\rho \circ \rho$ is the identity. It remains to show that $\rho(w) \in N$. Obviously $\rho(w)$ does not stay in $R$ (as $w$ does not). Hence, it suffices to show the following: if $\rho(w)=a_{1} \ldots a_{m} \mid b_{1} \ldots b_{m}$, then for each $i \in[n]$ and $1 \leq s<r$ we have $a_{r(i-1)+s} \geq a_{r(i-1)+s+1}$ and $b_{r(i-1)+s} \leq b_{r(i-1)+s+1}$. This is clearly true for every block $\{r(i-1)+s: 1 \leq s \leq r\}$ completely contained inside $w$ 's unchanged segment (i.e., $1, \ldots, t$ ) and inside $w$ 's modified segment (i.e., $t+1, \ldots, 2 m)$, given that it is true for $w$ and by the definition of $\rho$. There is still the case to handle where $t \in\{r(i-1)+s: 1 \leq s \leq r\}$. Here, it is true because as previously observed, $c_{t}=j+1$ if $t \leq m$ and $c_{t}=j$ otherwise. 


\section{The $d=2$ Case}

Our objective throughout this section is to initiate the study of pattern avoidance in ordered bipartite multi-graphs. We start by addressing the case of 2-regular bipartite multi-graphs where the pattern to be avoided is the one shown in Figure 5. In other words, we are now interested in the determination of the values of $g_{r}(n ; 2)$ for all choices of $r$ and $n$.

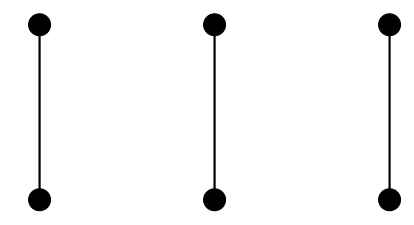

Figure 5: Multi-graph subpattern to avoid.

The walks encountered in Section 3 now become lattice paths in the plane - a subject intensively studied throughout combinatorics. Applying machinery for the enumeration of lattice paths in the plane we can determine the $\left|W_{I}^{*}(2, r, 2 r n ; T(\pi))\right|$ terms involved in the alternating sum of Theorem 7 and thus calculate $g_{r}(n ; 2)$. Indeed, since $W_{I}^{*}(2, r, 2 r n ; T(\pi))$ and $\widetilde{W}_{I}^{*}(2, r, 2 r n ; T(\pi))$ are in one to one correspondence, the reflection principle (see [EP04] for $r=1)$ illustrated in Figure 6 immediately leads to our next result.

Lemma 14. Let $\pi$ be a permutation of $\{1,2\}$. There is a bijection between walks $\widetilde{w}$ belonging to $\widetilde{W}_{I}^{*}(2, r, 2 r n ; T(\pi))$ and directed paths starting from $(0,0)$ satisfying the following conditions:

(i) each step of the path is of the form $(1, b)$ where $b$ is in $\{-r, \ldots, r\}$ and $b$ has the same parity as $r$,

(ii) the path ends in $(2 n, 0)$ if $\pi$ is the identity permutation, and in $(2 n,-2)$ otherwise.

Moreover, if $\widetilde{w}$ stays in the region $x_{1} \geq x_{2} \geq \ldots \geq x_{d} \geq 0$, then

(iii) the path stays in the positive quadrant $\mathbb{Z}_{\geq 0} \times \mathbb{Z}_{\geq 0}$,

(iv) when at height $h$, the path is restricted to making steps of the form $(1, b)$ provided $h \geq$ $(r-b) / 2$.

Enumeration of directed lattice paths satisfying conditions (i) and (ii) as in the preceding result is a well studied topic, see for example the unified coverage of the subject by Banderier and Flajolet [BF02]. Even the constraint imposed by (iii) of Lemma 14 is standard (see again [BF02]). In the special case that $r=2$, after re-scaling the $x_{2}$ axis by a factor of 2 , we get that the paths that satisfy conditions (i)-(iii) of Lemma 14 are the so called Motzkin paths [DS77] of length 2n, thus their cardinality are Motzkin numbers (EIS A000108). However, condition (iv) imposes an additional restriction, specifically that there are no horizontal steps at level 0. Still, for the $r=2$ case, enumeration of these restricted type of Motzkin paths is relatively simple and yield the sequence of Motzkin sums (EIS A005043). We are not aware of attempts to enumerate the restricted type of Motzkin paths we encounter when $r>2$. The 


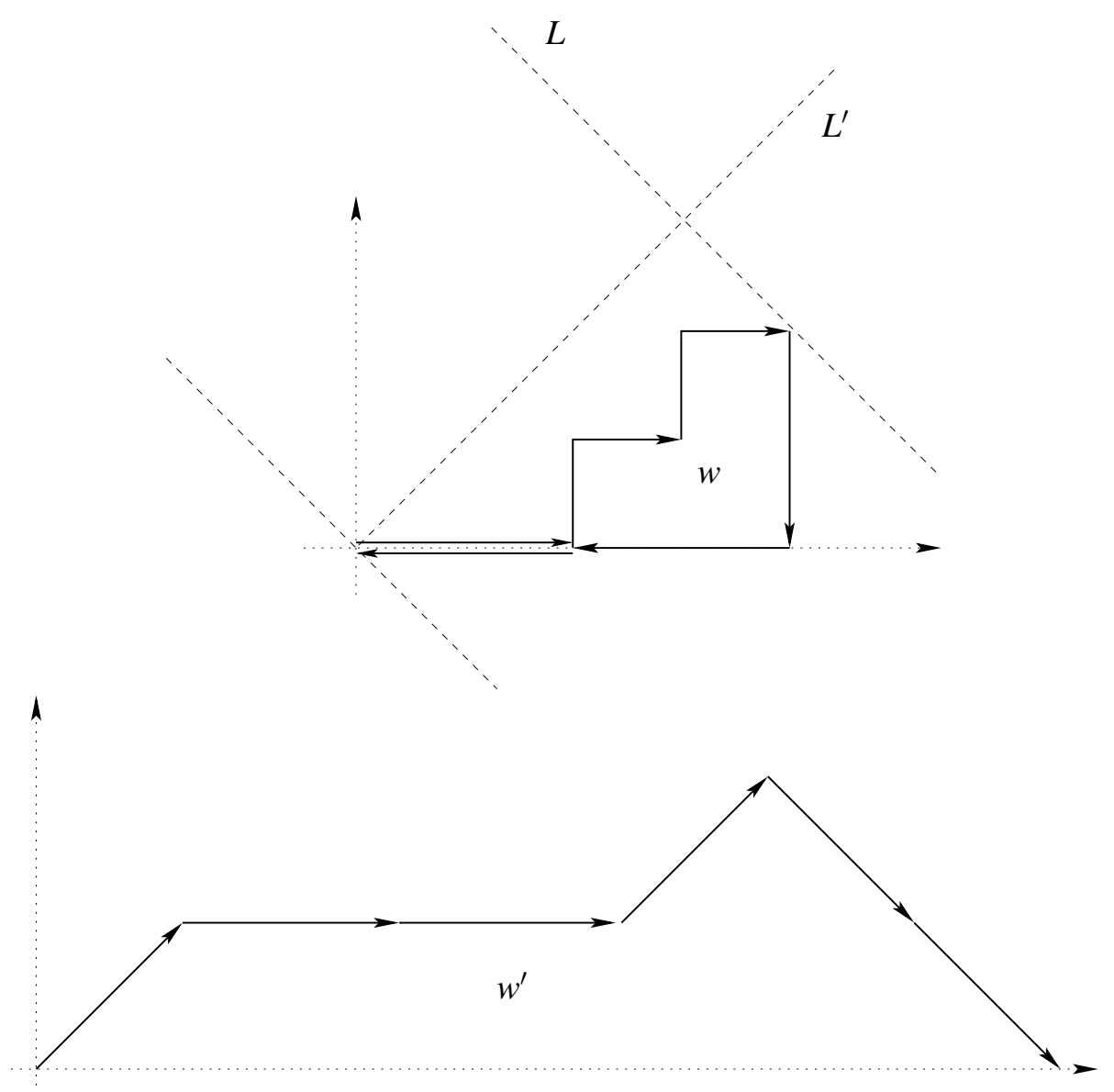

Figure 6: Walk $w \in W_{I}^{*}(2, r, 2 r n ; \overrightarrow{0})$ and walk $w^{\prime}$ obtained from $w$ by reflection over $L$ of the negative steps of $w$, followed by a reflection over $L^{\prime}$ of the derived walk.

techniques discussed in [BF02] do not seem to apply in this new setting. However, we do not need to enumerate such restricted type of Motzkin paths. Our real interest is the determination of $g_{r}(n ; 2)$. Here is where Theorem 7 turns out to be useful. The crucial observation is that Theorem 7 establishes that $g_{r}(n ; 2)$ is an alternating sum, and that the generating functions of the summands can be derived through the techniques laid out in [BF02]. Below we develop this line of argument.

Let $S_{r}$ be the set of elements of $\{-r, \ldots, r\}$ such that $b$ has the same parity as $r$. Consider the Laurent polynomial $P_{r}$ in $u, u^{-1}$ given by

$$
P_{r}(u)= \begin{cases}\sum_{j \in \mathcal{S}_{r}} u^{j / 2}, & \text { if } r \text { is even } \\ \sum_{j \in \mathcal{S}_{r}} u^{j}, & \text { if } r \text { is odd. }\end{cases}
$$

An immediate consequence of [BF02, Theorem 1] is the following result. 
Theorem 15. Let $c_{r}$ equal $r / 2$ if $r$ is even and $r$ otherwise. Let $u_{1}, \ldots, u_{c_{r}}$ be the small branches of the characteristic curve $1-z P_{r}(u)=0$. The generating function of directed paths starting at the origin, ending at altitude $h$, and relative to the set of steps $\left\{(1, b): b \in \mathcal{S}_{r}\right\}$ is an algebraic function such that

$$
V_{r, h}(z)= \begin{cases}z \sum_{j=1}^{c_{r}} \frac{u_{j}^{\prime}(z)}{u_{j}(z)}, & \text { if } h=0, \\ z \sum_{j=1}^{c_{r}} u_{j}^{\prime}(z), & \text { if } h=-2 .\end{cases}
$$

Theorem 7, Lemma 14, the fact that $W_{I}^{*}(2, r, 2 m ; T(\pi))$ and $\widetilde{W}_{I}^{*}(2, r, 2 m ; T(\pi))$ are in one to one correspondence, and Theorem 15 immediately yield the following result.

Theorem 16. Let $n$ and $r$ be positive integers. Then, $G_{r, 2}(z)=V_{r, 0}(z)-V_{r,-2}(z)$ is algebraic and $g_{r}(n ; 2)=\left[z^{2 n}\right] G_{r, 2}(z)$.

For instance, when $r=2$, then $c_{r}=1, u_{1}(z)=\left(1-\sqrt{1-2 z-3 z^{2}}\right) / 2 z$ and,

$$
G_{2,2}(z)=\frac{1+z-\sqrt{1-2 z-3 z^{2}}}{2 z(1+z)}=1+z^{2}+z^{3}+3 z^{4}+6 z^{5}+15 z^{6}+36 z^{7}+91 z^{8}+232 z^{9}+\ldots
$$

As already pointed out, the coefficients of $G_{2,2}(z)$ agree with the sequence EIS A005043. When $r=4$,

$$
\begin{aligned}
& u_{1}(z)=+z^{1 / 2}+\frac{1}{2} z+\frac{5}{8} z^{3 / 2}+z^{2}+\frac{231}{128} z^{5 / 2}+3 z^{6}+\frac{5501}{1024} z^{7 / 2}+10 z^{4} \ldots \\
& u_{2}(z)=-z^{1 / 2}+\frac{1}{2} z-\frac{5}{8} z^{3 / 2}+z^{2}-\frac{231}{128} z^{5 / 2}+3 z^{6}-\frac{5501}{1024} z^{7 / 2}+10 z^{4} \ldots
\end{aligned}
$$

Thus,

$$
G_{4,2}(z)=1+z^{2}+z^{3}+5 z^{4}+16 z^{5}+65 z^{6}+260 z^{7}+1085 z^{8}+4600 z^{9}+19845 z^{10} \ldots
$$

Starting with the second coefficient of $G_{4,2}(z)$ yields the same sequence as obtained by dropping the first two terms of sequence EIS A007043.

When $r=1$, the coefficients of $G_{1,2}(z)$ count the number of 123 avoiding permutations which by a classical result is known to be the $n$-th Catalan number with ordinary generating function:

$$
G_{1,2}(z)=\frac{1-\sqrt{1-4 z}}{2 z^{2}}=1+z^{2}+2 z^{4}+5 z^{6}+14 z^{8}+42 z^{10}+132 z^{12}+429 z^{14}+1430 z^{16}+\ldots
$$

The next odd $r$ case is:

$$
G_{3,2}(z)=1+z^{2}+4 z^{4}+34 z^{6}+364 z^{8}+4269 z^{10}+52844 z^{12}+679172 z^{14}+8976188 z^{16} \ldots
$$




\section{The Arbitrary $d$ and $r$ Case}

In this section we derive an expression that enables the computation (for small values of $d$ and $r$ ) of the quantities $\left|W_{I}^{*}(d, r, 2 r n ; T(\pi))\right|$ involved in the identity of Theorem 7 . Thence, in addition to the values of $g_{r}(n ; 2)$ computed in the previous section, we will now be able to determine $g_{r}(n ; d)$ for small values of $r$ and $d>2$.

It will be convenient to denote by $W^{*}\left(d, r, m^{+}, m^{-} ; \vec{p}\right)$ the set of walks of $W^{*}(d, r, m ; \vec{p})$ with $m^{+}$positive steps and $m^{-}$negative steps, where $m=m^{+}+m^{-}$. We will abide by a similar convention when referring to $W_{I}^{*}(d, r, m ; \vec{p})$. We denote by $w_{I}^{*}\left(d, r, m^{+}, m^{-} ; \vec{p}\right)$ the cardinality of $W_{I}^{*}\left(d, r, m^{+}, m^{-} ; \vec{p}\right)$. Henceforth, we will assume $m^{+}$and $m^{-}$are multiples of $r$, specifically that $m^{+}=r n^{+}$and $m^{-}=r n^{-}$.

Let $\Omega \subseteq[d]^{r}$ be the family of vectors $\left(v_{1}, \ldots, v_{r}\right)$ such that $v_{1} \geq v_{2} \geq \ldots \geq v_{r}$. Also let $\bar{\Omega}=$ $[d]^{r} \backslash \Omega$. The set $\Omega$ represents the collection of valid $r$-factors of walks in $W_{I}^{*}\left(d, r, m^{+}, m^{-} ; \vec{p}\right)$.

For $f: A \subseteq U \rightarrow \bar{\Omega}$ and $g: B \subseteq V \rightarrow \bar{\Omega}$ let $W^{*}\left(d, r, f, g ; m^{+}, m^{-} ; \vec{p}\right)$ be the collection of walks in $W^{*}\left(d, r, m^{+}, m^{-} ; \vec{p}\right)$ whose $u$-th positive $r$-factor is $f(u)$ when $u \in \operatorname{Dom}(f)$ and similarly its $v$-th negative $r$-factor is given by $g(v)$ provided $v \in \operatorname{Dom}(g)$. Formally, $W^{*}\left(d, r, f, g ; m^{+}, m^{-} ; \vec{p}\right)$ denotes the set of walks $a_{u_{1}^{1}} \ldots a_{u_{n^{+}}^{r}} \mid b_{v_{1}^{1}} \ldots b_{v_{n^{-}}^{r}}$ in $W^{*}\left(d, r, m^{+}, m^{-} ; \vec{p}\right)$ such that

- if $u \in A$, then $\left(a_{u^{1}}, \ldots, a_{u^{r}}\right)=f(u)$,

- if $v \in B$, then $\left(b_{v^{1}}, \ldots, b_{v^{r}}\right)=g(v)$.

Note that the definition of $W^{*}\left(d, r, f, g ; m^{+}, m^{-} ; \vec{p}\right)$ makes sense even when the domains of $f$ or $g$ are the empty set. Henceforth, we denote by $w^{*}\left(d, r, f, g ; m^{+}, m^{-} ; \vec{p}\right)$ the cardinality of $W^{*}\left(d, r, f, g ; m^{+}, m^{-} ; \vec{p}\right)$.

For a positive integer $k$, let $\mathcal{F}_{k}$ denote the collection of mappings $f: A \subseteq U \rightarrow \bar{\Omega}$ such that $|A|=k$. For $l \in \mathbb{N}, l>0$, define analogously the collection $\mathcal{G}_{l}$ of mappings $g: B \subseteq V \rightarrow \bar{\Omega}$ such that $|B|=l$. Let $\mathcal{F}_{0}=\mathcal{G}_{0}=\{\emptyset\}$, where $\emptyset$ denotes the function that goes from the empty domain into $\bar{\Omega}$.

We start by establishing a relation between the terms $w_{I}^{*}\left(d, r, m^{+}, m^{-} ; \vec{p}\right)$ and the somewhat more manageable quantities $w^{*}\left(d, r, f, g ; m^{+}, m^{-} ; \vec{p}\right)$. The relation makes use of the standard convention of denoting $a(a-1) \cdots(a-b+1)$ by $(a)_{b}$ where $a$ and $b$ are non-negative integers.

Lemma 17. For every $\vec{p} \in \mathbb{Z}^{d}$, and $m^{+}, m^{-} \in r \mathbb{N}$,

$$
\begin{gathered}
\frac{w_{I}^{*}\left(d, r, m^{+}, m^{-} ; \vec{p}\right)}{m^{+} ! m^{-} !}=\sum_{k, l \geq 0}(-1)^{k+l} \sum_{f \in \mathcal{F}_{k}} \sum_{g \in \mathcal{G}_{l}} \frac{w^{*}\left(d, r, f, g ; m^{+}, m^{-} ; \vec{p}\right)}{m^{+} ! m^{-} !} \\
=\sum_{k, l \geq 0} \frac{(-1)^{k+l}}{\left(m^{+}\right)_{r k}\left(m^{-}\right)_{r l}} \sum_{f \in \mathcal{F}_{k}} \sum_{g \in \mathcal{G}_{l}} \frac{w^{*}\left(d, r, f, g ; m^{+}, m^{-} ; \vec{p}\right)}{\left(m^{+}-r k\right) !\left(m^{-}-r l\right) !} .
\end{gathered}
$$

Proof. We will prove only the first equality. The other one is elementary. First observe that

$$
\begin{aligned}
& W_{I}^{*}\left(d, r, m^{+}, m^{-} ; \vec{p}\right)= \\
& \quad W^{*}\left(d, r, m^{+}, m^{-} ; \vec{p}\right) \backslash\left(\bigcup_{f \in \mathcal{F}_{1}} W^{*}\left(d, r, f, \emptyset ; m^{+}, m^{-} ; \vec{p}\right) \cup \bigcup_{g \in \mathcal{G}_{1}} W^{*}\left(d, r, \emptyset, g ; m^{+}, m^{-} ; \vec{p}\right)\right) .
\end{aligned}
$$


Hence, by the Principle of Inclusion-Exclusion,

$$
w_{I}^{*}\left(d, r, m^{+}, m^{-} ; \vec{p}\right)=\sum_{k \geq 0} \sum_{l \geq 0}(-1)^{k+l} \sum_{f \in \mathcal{F}_{k}} \sum_{g \in \mathcal{G}_{l}} w^{*}\left(d, r, f, g ; m^{+}, m^{-} ; \vec{p}\right) .
$$

The desired conclusion follows immediately.

We now seek to express the quantity $w^{*}\left(d, r, f, g ; m^{+}, m^{-} ; \vec{p}\right)$ as a term that only involves expressions that count certain type of one-dimensional walks. Note that there are one-dimensional walks in $\mathbb{Z}$ from the origin to $p \in \mathbb{Z}$ with $m^{+}$positive steps preceding $m^{-}$negative steps if and only if $m^{+}-m^{-}=p$. Moreover, there is at most one such walk which we denote by $w\left(m^{+}, m^{-} ; p\right)$.

Henceforth we rely on Knuth's championed form of Iverson's convention [Knu92]:

$$
\left[m^{+}-m^{-}=p\right]= \begin{cases}1, & \text { if } m^{+}-m^{-}=p, \\ 0, & \text { otherwise }\end{cases}
$$

Also, if $\varphi$ takes values in $[d]^{r}$ we let $\varphi_{s}$ denote the total number of coordinates in $\varphi(x)$ equal to $s$ as $x$ varies over $\varphi$ 's domain, i.e.

$$
\varphi_{s}=\sum_{x \in \operatorname{Dom}(\varphi)}\left|\left\{t \in[r]:(\varphi(x))_{t}=s\right\}\right|
$$

Lemma 18. For every $k, l \in \mathbb{N}, f \in \mathcal{F}_{k}$ and $g \in \mathcal{G}_{l}, \vec{p}=\left(p_{1}, \ldots, p_{d}\right) \in \mathbb{Z}^{d}$, and $m^{+}, m^{-} \in r \mathbb{N}$,

$$
\frac{w^{*}\left(d, r, f, g ; m^{+}, m^{-} ; \vec{p}\right)}{\left(m^{+}-r k\right) !\left(m^{-}-r l\right) !}=\sum_{\substack{m_{1}^{+}+\ldots+m_{d}^{+}=m^{+} \\ m_{i}^{+} \geq f_{i}, i \in[d] \quad}} \sum_{\substack{m_{1}^{-}+\ldots+m_{d}^{-}=m^{-} \\ m_{i}^{-} \geq g_{i}, i \in[d]}} \prod_{i=1}^{d} \frac{\left[m_{i}^{+}-m_{i}^{-}=p_{i}\right]}{\left(m_{i}^{+}-f_{i}\right) !\left(m_{i}^{-}-g_{i}\right) !}
$$

Proof. Map each walk $w$ in $W^{*}\left(d, r, f, g ; m^{+}, m^{-} ; \vec{p}\right)$ to $d$ one-dimensional walks $\left(w_{1}, \ldots, w_{d}\right)$ where $w_{i}$ corresponds to the steps in dimension $i$. Consider the $d$-tuple of one-dimensional walks $\left(w_{1} \ldots, w_{d}\right)$ such that $w_{i}=w\left(m_{i}^{+}, m_{i}^{-} ; p_{i}\right)$, where $m^{+}=\sum_{i} m_{i}^{+}$and $m^{-}=\sum_{i} m_{i}^{-}$. We claim that $\left(w_{1}, \ldots, w_{d}\right)$ is the image through the aforementioned mapping of

$$
\left(\begin{array}{c}
m^{+}-r k \\
m_{1}^{+}-f_{1}, \ldots, m_{d}^{+}-f_{d}
\end{array}\right)\left(\begin{array}{c}
m^{-}-r l \\
m_{1}^{-}-g_{1}, \ldots, m_{d}^{-}-g_{d}
\end{array}\right)
$$

elements of $W^{*}\left(d, r, f, g ; m^{+}, m^{-} ; \vec{p}\right)$. Indeed, consider a preimage in $W^{*}\left(d, r, f, g ; m^{+}, m^{-} ; \vec{p}\right)$ of $\left(w_{1}, \ldots, w_{d}\right)$, say $w=a_{u_{1}^{1}} \cdots a_{u_{n^{+}}^{r}} \mid b_{v_{1}^{1}} \cdots b_{v_{n^{-}}^{r}}$. Clearly, if $f$ is defined over $u$, then it must hold that $f(u) \in \bar{\Omega}$. Similarly, if $g$ is defined over $v$, then $g(v) \in \bar{\Omega}$. It follows that $w=$ $a_{u_{1}^{1}} \ldots a_{u_{n^{+}}^{r}} \mid b_{v_{1}^{1}} \ldots b_{v_{n^{-}}^{r}}$ is a preimage of $\left(w_{1}, \ldots, w_{d}\right)$ if and only if for each $i \in[d]$ one finds among $\left\{a_{u^{j}}: u \notin \operatorname{Dom}(f), j \in[r]\right\}$ and $\left\{b_{v^{j}}: v \notin \operatorname{Dom}(g), j \in[r]\right\}$ exactly $m_{i}^{+}-f_{i}$ and $m_{i}^{-}-g_{i}$ elements taking the value $i$, respectively. This completes the proof of the claim.

Putting things together yields the sought after result. 


\begin{tabular}{|c|r|r|r|r|r|r|}
\hline$(d, n)$ & 2 & \multicolumn{1}{|c|}{3} & \multicolumn{1}{c|}{4} & \multicolumn{1}{c|}{5} & \multicolumn{1}{c|}{6} & \multicolumn{1}{c|}{7} \\
\hline 2 & 3 & 15 & 91 & 603 & 4,213 & 30,537 \\
3 & 3 & 21 & 258 & 4,171 & 78,021 & $1,604,777$ \\
4 & 3 & 21 & 282 & 6,090 & 179,371 & \\
5 & 3 & 21 & 282 & 6,210 & & \\
\hline
\end{tabular}

Table 1: Values of $g_{2}(n ; d)$ for small values of $d$ and $n$.

Corollary 19. For every $\vec{p}=\left(p_{1}, \ldots, p_{d}\right) \in \mathbb{Z}^{d}$ and $m^{+}, m^{-} \in r \mathbb{N}$,

$$
\begin{aligned}
& \frac{w_{I}^{*}\left(d, r, m^{+}, m^{-} ; \vec{p}\right)}{m^{+} ! m^{-} !}= \\
& \sum_{k, l \geq 0} \frac{(-1)^{k+l}\left(\begin{array}{c}
m^{+} / r \\
k
\end{array}\right)\left(\begin{array}{c}
m^{-} / r \\
l
\end{array}\right)}{\left(m^{+}\right)_{r k}\left(m^{-}\right)_{r l}} \sum_{\substack{\varphi:[k] \rightarrow \bar{\Omega}, \phi:[l] \rightarrow}} \sum_{\substack{m_{1}^{+}+\ldots+m_{d}^{+}=m^{+}, m_{i}^{+} \geq \varphi_{i}, i \in[d]}} \sum_{\substack{m_{1}^{-}+\ldots+m_{d}^{-}=m^{-}, m_{i}^{-} \geq \phi_{i}, i \in[d]}} \prod_{i=1}^{d} \frac{\left[m_{i}^{+}-m_{i}^{-}=p_{i}\right]}{\left(m_{i}^{+}-\varphi_{i}\right) !\left(m_{i}^{-}-\phi_{i}\right) !} .
\end{aligned}
$$

Proof. Follows directly from Lemma 17, Lemma 18 and the fact that the expression

$$
\sum_{\substack{m_{1}^{+}+\ldots+m_{d}^{+}=m^{+}, m_{i}^{+} \geq f_{i}, i \in[d]}} \sum_{\substack{m_{1}^{-}+\ldots+m_{d}^{-}=m^{-} \\ m_{i}^{-} \geq g_{i}, i \in[d]}} \prod_{i=1}^{d} \frac{\left[m_{i}^{+}-m_{i}^{-}=p_{i}\right]}{\left(m_{i}^{+}-f_{i}\right) !\left(m_{i}^{-}-g_{i}\right) !}
$$

does not depend on what the domains of $f \in \mathcal{F}_{k}$ and $g \in \mathcal{G}_{l}$ are, but only in their size.

In this work, we are only interested in counting lattice walks that start at the origin and end at some Toeplitz point. All these walks must necessarily have the same number of positive and negative steps. This explains why we are solely interested in applying Corollary 19 when $\mathrm{m}^{+}=$ $m^{-}=r n$. In such cases, the number of non-zero summands of the 2 innermost summations in (1) is at most $\left(\begin{array}{c}r n+d-1 \\ d-1\end{array}\right)$. Furthermore, the 3 innermost summations depend on $\phi$ and $\varphi$ only through the $\phi_{i}$ 's and $\varphi_{i}$ 's which are completely determined by the size of the preimages through $\phi$ and $\varphi$ of each element in $\bar{\Omega}$. Thus, the 3 innermost summations in (1) can be computed summing at most

$$
\left(\begin{array}{c}
k+|\bar{\Omega}|-1 \\
|\bar{\Omega}|-1
\end{array}\right)\left(\begin{array}{c}
l+|\bar{\Omega}|-1 \\
|\bar{\Omega}|-1
\end{array}\right)\left(\begin{array}{c}
r n+d-1 \\
d-1
\end{array}\right)
$$

non-zero terms, each of which consists in $O(d)$ products of factorial terms. Note that $|\bar{\Omega}|=$ $2^{r}-(r+1)$ when $d=2,|\bar{\Omega}|=d(d-1) / 2$ when $r=2$, and in general $|\bar{\Omega}| \leq d^{r}$. Thus, for fixed values of $d$ and $r$, we can determine the values of $\left|W_{I}^{*}(d, r, 2 r n ; T(\pi))\right|$ and $g_{r}(n ; d)$ by performing some polynomial in $n$ number of operations. However, the degree of the polynomial involved is polynomial in $d$ and exponential in $r$, making the calculations prohibitively costly except for very small values of $d$ and $r$. Table 1 shows some values of $g_{r}(n ; d)$ for $r=3$ calculated by means of Corollary 19 and Theorem 7 .

The right hand side of the expression in Corollary 19 can be written in terms of primitives and derivatives of more complicated expressions. This will allow us to derive an elegant formula 
for the left hand side expression of (1). Let $\partial x$ denote the partial derivative with respect to $x$. The $t$ times successive application of $\partial x$ will be denoted $\partial^{t} x$. Similarly, let $\partial^{-1} x$ denote the inverse operator of $\partial x$ (in other words, the operator returning a primitive with respect to $x$ ). Also, we denote by $\partial^{-t} x$ the $t$ times repeated application of $\partial^{-1} x$.

Corollary 20. For $p \in \mathbb{Z}$ and $k, l \in \mathbb{N}$, let

$$
A^{k, l}(p)=\sum_{s^{+} \geq k, s^{-} \geq l} \frac{\left[s^{+}-s^{-}=p\right]}{\left(s^{+}-k\right) !\left(s^{-}-l\right) !}\left(y_{+}^{1 / r} x_{+}\right)^{s^{+}}\left(y_{-}^{1 / r} x_{-}\right)^{s^{-}} .
$$

Then, for every $\vec{p}=\left(p_{1}, \ldots, p_{d}\right) \in \mathbb{Z}^{d}$,

$$
\begin{aligned}
& \sum_{m^{+}, m^{-} \in r \mathbb{N}} \frac{w_{I}^{*}\left(d, r, m^{+}, m^{-} ; \vec{p}\right)}{m^{+} ! m^{-} !} x_{+}^{m^{+}} x_{-}^{m^{-}}= \\
& \left.\sum_{k, l \geq 0} \frac{(-1)^{k+l}}{k ! l !} \sum_{\substack{\varphi:[k] \rightarrow \bar{\Omega}, \phi:[l] \rightarrow \bar{\Omega}}}\left(\left.\partial^{-r k} z_{+} \partial^{-r l} z_{-}\left(\partial^{k} y_{+} \partial^{l} y_{-} \prod_{i=1}^{d} A^{\varphi_{i}, \phi_{i}}\left(p_{i}\right)\right)\right|_{\substack{y_{+}=z_{+}^{r} \\
y_{-}=z_{-}^{+}}}\right)\right|_{\substack{z_{+}=1, z_{-}=1}}
\end{aligned}
$$

restricted to the powers of both $x_{+}$and $x_{-}$divisible by $r$.

Proof. First, note that for $\varphi:[k] \rightarrow \bar{\Omega}$ and $\phi:[l] \rightarrow \bar{\Omega}$,

$$
\begin{aligned}
& \prod_{i=1}^{d} A^{\varphi_{i}, \phi_{i}}\left(p_{i}\right)= \\
& \sum_{m^{+}, m^{-} \geq 0}\left(\sum_{\substack{m_{1}^{+}+\ldots+m_{d}^{+}=m^{+}, m_{1}^{-}+\ldots+m_{d}^{-}=m^{-}, i=1 \\
m_{i}^{+} \geq \varphi_{i}}} \prod_{m_{i}^{-} \geq \phi_{i}}^{d} \frac{\left[m_{i}^{+}-m_{i}^{-}=p_{i}\right]}{\left(m_{i}^{+}-\varphi_{i}\right) !\left(m_{i}^{-}-\phi_{i}\right) !}\right)\left(y_{+}^{1 / r} x_{+}\right)^{m^{+}}\left(y_{-}^{1 / r} x_{-}\right)^{m^{-}} .
\end{aligned}
$$

If we denote by $\%$ the expression in between parentheses above, it follows immediately that

$$
\left.\partial^{k} y_{+} \partial^{l} y_{-} \prod_{i=1}^{d} A^{\varphi_{i}, \phi_{i}}\left(p_{i}\right)\right|_{\substack{y_{+}=z_{+}^{r} \\ y_{-}=z_{-}^{r}}}=\sum_{m^{+}, m^{-} \geq 0}(\%)\left(m^{+} / r\right)_{k}\left(m^{-} / r\right){ }_{l} z_{+}^{m^{+}-r k} z_{-}^{m^{-}-r l} x_{+}^{m^{+}} x_{-}^{m^{-}},
$$

and

$$
\begin{gathered}
\left.\left(\left.\partial^{-r k} z_{+} \partial^{-r l} z_{-}\left(\partial^{k} y_{+} \partial^{l} y_{-} \prod_{i=1}^{d} A^{\varphi_{i}, \phi_{i}}\left(p_{i}\right)\right)\right|_{\substack{y_{+}=z_{+}^{r} \\
y_{-}=z_{-}^{+}}}\right)\right|_{\substack{z_{+}=1, z_{-}=1}}= \\
\sum_{m^{+}, m^{-} \geq 0}(\%) \frac{\left(m^{+} / r\right)_{k}\left(m^{-} / r\right)_{l}}{\left(m^{+}\right)_{r k}\left(m^{-}\right)_{r l}} x_{+}^{m^{+}} x_{-}^{m_{-}^{-}} .
\end{gathered}
$$


Hence,

$$
\begin{gathered}
\left.\sum_{k, l \geq 0} \frac{(-1)^{k+l}}{k ! l !} \sum_{\substack{\varphi:[k] \rightarrow \bar{\Omega}, \phi:[l] \rightarrow \bar{\Omega}}}\left(\left.\partial^{-r k} z_{+} \partial^{-r l} z_{-}\left(\partial^{k} y_{+} \partial^{l} y_{-} \prod_{i=1}^{d} A^{\varphi_{i}, \phi_{i}}\left(p_{i}\right)\right)\right|_{\substack{y_{+}=z_{+}^{r} \\
y_{-}=z_{-}^{r}}}\right)\right|_{\substack{z_{+}=1, z_{-}=1}}= \\
\sum_{m^{+}, m^{-} \geq 0} \sum_{k, l \geq 0} \frac{(-1)^{k+l}}{k ! l !} \frac{\left(m^{+} / r\right)_{k}\left(m^{-} / r\right)_{l}}{\left(m^{+}\right)_{r k}\left(m^{-}\right)_{r l}} \sum_{\substack{\varphi:[k] \rightarrow \bar{\Omega}, \phi:[l] \rightarrow \bar{\Omega}}}(\%) x_{+}^{m^{+}} x_{-}^{m^{-}} .
\end{gathered}
$$

To conclude the proof, observe that by Corollary 19, when $m^{+}, m^{-} \in r \mathbb{N}$ the coefficient of $x_{+}^{m^{+}} x_{-}^{m^{-}}$in the right hand side of the above identity is equal to $w_{I}^{*}\left(d, r, m^{+}, m^{-} ; \vec{p}\right) /\left(m^{+} ! m^{-} !\right)$.

Let

$$
C(p ; a, b)=\sum_{s^{+}, s^{-} \geq 0} \frac{\left[s^{+}-s^{-}=p\right]}{s^{+} ! s^{-} !}\left(y_{+}^{1 / r} x_{+} a\right)^{s^{+}}\left(y_{-}^{1 / r} x_{-} b\right)^{s^{-}},
$$

and observe that

$$
\prod_{i=1}^{d} A^{\varphi_{i}, \phi_{i}}\left(p_{i}\right)=\left.\partial^{\varphi_{1}} a_{1} \partial^{\phi_{1}} b_{1} \ldots \partial^{\varphi_{d}} a_{d} \partial^{\phi_{d}} b_{d} \prod_{i=1}^{d} C\left(p_{i} ; a_{i}, b_{i}\right)\right|_{a_{i}=b_{i}=1, i \in[d]} .
$$

If we now define the differential operator

$$
\begin{aligned}
& \mathcal{D}(f)= \\
& \left.\sum_{k, l \geq 0} \frac{(-1)^{k+l}}{k ! l !} \sum_{\substack{\varphi:[k] \rightarrow \bar{\Omega}, \phi:[l] \rightarrow \bar{\Omega}}}\left(\left.\partial^{-r k} z_{+} \partial^{-r l} z_{-}\left(\left.\partial^{k} y_{+} \partial^{l} y_{-}\left(\prod_{i=1}^{d} \partial^{\varphi_{i}} a_{i} \partial^{\phi_{i}} b_{i} f\right)\right|_{\substack{a_{i}=1, b_{i}=1}}\right)\right|_{\substack{y_{+}=z_{+}^{r} \\
y_{-}=z_{-}^{r}}}\right)\right|_{\substack{z_{+}=1, z_{-}=1}},
\end{aligned}
$$

we obtain the following result.

Theorem 21. Let $|M|$ denote the determinant of $M$. Then,

$$
\sum_{n \geq 0} \frac{g_{r}(n ; d)}{(r n) !^{2}} x_{+}^{r n} x_{-}^{r n}=\mathcal{D}\left(\left|C\left(i-j, a_{i}, b_{i}\right)_{i, j=1, \ldots, d}\right|\right)
$$

restricted to the powers of both $x_{+}$and $x_{-}$divisible by $r$.

Proof. Since a walk in $W_{I}^{*}(d, r, 2 r n ; T(\pi))$ has exactly $r n$ positive and negative steps, we get that $\left|W_{I}^{*}(d, r, 2 r n ; T(\pi))\right|=w_{I}^{*}(d, r, r n, r n ; T(\pi))$. Also, we get that $w_{I}^{*}\left(d, r, m^{+}, m^{-} ; T(\pi)\right)=0$ unless $m^{+}=m^{-}$. Thus, by Theorem 7 and Corollary 20

$$
\begin{aligned}
\sum_{n \geq 0} \frac{g_{r}(n ; d)}{(r n) !^{2}} x_{+}^{r n} x_{-}^{r n} & =\sum_{\pi \in S_{d}} \operatorname{sign}(\pi) \sum_{m^{+}, m^{-} \in r \mathbb{N}} \frac{w_{I}^{*}\left(d, r, m^{+}, m^{-} ; T(\pi)\right)}{m^{+} ! m^{-} !} x_{+}^{m^{+}} x_{-}^{m^{-}} \\
& =\mathcal{D}\left(\left|C\left(i-j, a_{i}, b_{i}\right)_{i, j=1, \ldots, d}\right|\right)
\end{aligned}
$$

restricted to the powers of both $x_{+}$and $x_{-}$divisible by $r$. 
Finally, consder the $r=1$ case. Since $\Omega=[d]$, then $\bar{\Omega}=\emptyset$. Thus, $\mathcal{F}_{k}=\mathcal{G}_{l}=\emptyset$ for all positive integers $k$ and $l$. Moreover,

$$
C(p ; a, b)= \begin{cases}\sum_{s \geq 0} \frac{1}{(s+p) ! s !}\left(y_{+} x_{+} a\right)^{s+p}\left(y_{-} x_{-} b\right)^{s}, & \text { if } p \geq 0 \\ \sum_{s \geq 0} \frac{1}{s !(s+p) !}\left(y_{+} x_{+} a\right)^{s}\left(y_{-} x_{-} b\right)^{s+p}, & \text { otherwise. }\end{cases}
$$

It follows that for $r=1$, the differential operator $\mathcal{D}(f)$ reduces to evaluating $f$ at $a_{i}=b_{i}=1$ for all $i \in[d]$ and $y_{+}=z_{+}=y_{-}=y_{+}=1$. Thus, Gessel's identity is recovered by setting $r=1$ and $x_{+}=x_{-}=x$ in Theorem 21 .

\section{References}

[AD99] D. Aldous and P. Diaconis. Longest increasing subsequences: from patience sorting to the Baik-Deift-Johansson theorem. Bull. of the AMS, 36(4):413-432, 1999.

[B0́4] M. Bóna. Combinatorics of Permutations. Discrete Mathematics and its Applications. Chapman \& Hall/CRC, 2004.

[BB67] R. Baer and P. Brock. Natural sorting over permutation spaces. Math. Comp., pages 385-410, 1967.

[BDJ99] J. Baik, P. Deift, and K. Johansson. On the distribution of the length of the longest increasing subsequence of random permutations. J. Amer. Math. Soc., 12:1119$1178,1999$.

[BF02] C. Banderier and P. Flajolet. Basic analytic combinatorics of directed lattice paths. Theor. Comput. Sci., 281(1-2):37-80, 2002.

[BM06] M. Bousquet-Melou. Discrete excursions. Sémin. Lothar. Comb., 57, 2006.

[Bol85] B. Bollobás. Random Graphs. Academic Press, 1985.

[BR01] J. Baik and E.M. Rains. Symmetrised random permutations. In MSRI volume 40: Random Matrix Models and their Applications, pages 1-19, 2001.

$\left[\mathrm{CDD}^{+}\right.$07] W.Y.C. Chen, E.Y. Deng, R.R.X. Du, R.P. Stanley, and C.H. Yan. Crossings and nestings of matchings and partitions. Trans. Amer. Math. Soc, 359(4):1555-1575, 2007.

[de 07] A. de Mier. $k$-noncrossing and $k$-nonnesting graphs and fillings of Ferrers diagrams. Combinatorica, 27:699-720, 2007.

[DS77] R. Donaghey and L. W. Shapiro. Motzkin numbers. J. Combin. Theory Ser. A, 23:291-301, 1977.

[EP04] S. Elizalde and I. Pak. Bijections for refined restricted permutations. J. Comb. Theory, Ser. A, 105:207-219, 2004.

[ES35] P. Erdős and G. Szekeres. A combinatorial problem in geometry. Compositio Math., 2:463-470, 1935. 
[Fu197] W. Fulton. Young Tableaux, volume 35 of Student Texts. London Mathematical Society, 1997.

[Ges90] I. Gessel. Symmetric functions and P-recursiveness. J. Comb. Theory, Ser. A, 53(2):257-285, 1990.

[GTW01] J. Gravner, C. Tracy, and H. Widom. Limit theorems for height fluctuations in a class of discrete space time growth models. J. Stat. Phys., 102:1085-1132, 2001.

[GWW98] I. Gessel, J. Weinstein, and H. Wilf. Lattice walks in $\mathbb{Z}^{d}$ and permutations with no long ascending subsequences. Electron. J. of Combin., 5(2):R2, 1998.

[Ham72] J. M. Hammersley. A few seedlings of research. In Proc. Sixth Berkeley Sympos. Math. Stat. Prob., pages 345-394, Berkeley, CA, 1972. Universisty of California Press.

[Joh00] K. Johannson. Shape fluctuations and random matrices. Commun. Math. Phys., 209:437-476, 2000.

[Kin73] J. F. C. Kingman. Subadditive ergodic theory. Ann. Probab., 1(6):883-909, 1973.

[Knu92] D. Knuth. Two notes on notation. Amer. Math. Monthly, 99(5):403-422, 1992.

[LS77] B. Logan and L. Shepp. A variational problem for random Young tableaux. Adv. in Math., 26:206-222, 1977.

[Oko00] A. Okounkov. Random matrices and random permutations. Internat. Math. Res. Notices, pages 1043-1095, 2000.

[Sch61] C. Schensted. Longest increasing and decreasing subsequences. Canad. J. Math., 13:179-191, 1961.

[Sep77] T. Seppäläinen. Exact limiting shape for a simplified model of first-passage percolation on the plane. Ann. Probab., 26(3):1232-1250, 1977.

[Sta02] R. Stanley. Recent progress in algebraic combinatorics. Bull. of the AMS, 40(1):5568, 2002.

[Ula61] S. Ulam. Monte Carlo calculations in problems of mathematical physics. In Modern Mathematics for the Engineers, pages 261-281. McGraw-Hill, 1961.

[VK77] A. Vershik and S. Kerov. Asymptotics of the Plancherel measure of the symmetric group and the limiting form of Young tableaux. Dokl. Akad. Nauk SSSR, 233:1024$1028,1977$. 